\title{
Kernos
}

Revue internationale et pluridisciplinaire de religion grecque antique

16 | 2003

Varia

\section{Chanter les dieux grecs}

Ellen Van Keer

(2) OpenEdition

Journals

Édition électronique

URL : http://journals.openedition.org/kernos/837

DOI : $10.4000 /$ kernos.837

ISSN : 2034-7871

Éditeur

Centre international d'étude de la religion grecque antique

Édition imprimée

Date de publication : 1 janvier 2003

Pagination : 357-363

ISSN : 0776-3824

Référence électronique

Ellen Van Keer, «Chanter les dieux grecs », Kernos [En ligne], 16 | 2003, mis en ligne le 14 avril 2011, consulté le 22 septembre 2020. URL : http://journals.openedition.org/kernos/837 ; DOI : https:// doi.org/10.4000/kernos.837 


\title{
Revue des Livres
}

\section{Compte rendu critique}

\author{
Chanter les dieux grecs ${ }^{1}$
}

par Ellen VAN KEER

L'étude des religions anciennes manifeste un épanouissement permanent, mais ce développement ne touche pas à tous les aspects des comportements, des expériences et des représentations religieuses de l'antiquité. En histoire des religions, la dimension musicale des phénomènes religieux n'est guère abordée. Elle est davantage traitée sous l'égide de la musicologie qui «monopolise » l'étude de la musique. Mais, si l'on soustrait les aspects musicaux des mythes, des dieux, des cultes et des rites du champ de l'histoire des religions, l'ampleur de ces phénomènes musicaux comme des phénomènes religieux auxquels ils sont liés risque d'échapper à la compréhension. En effet, à cause des cloisonnements académiques, tant la dimension musicale de la vie religieuse que la dimension religieuse de la vie musicale et les relations étroites entre les deux restent hors d'atteinte de la recherche traditionnelle. C'est pourquoi Pierre Brulé, historien de la religion grecque, et Christophe Vendries, archéologue de la musique, ont, avec clairvoyance, organisé ce colloque considérant les interactions entre la musique et la religion dans l'antiquité selon un double regard musical et religieux. Nous ne pouvons que saluer la publication qui suit cette heureuse initiative.

La problématique a produit une multiplicité de thématiques, de questionnements et d'approches. Suivant leur filiation académique ou leurs intérêts personnels, les auteurs ont favorisé les sources textuelles, visuelles ou matérielles; ils ont abordé la culture grecque, étrusque ou romaine; ils ont porté l'accent sur les pratiques cultuelles, les représentations mythologiques ou les expériences musicales etc. - la liste est longue. Cette variété démontre la richesse et la complexité du sujet de ces Actes, qui n'ont nullement la prétention de l'épuiser ni de constituer un aboutissement définitif. Il s'agit plutôt d'une première introduction à une problématique encore peu explorée et, de ce point de vue, le volume est très réussi. Sur le plan du contenu et de la méthodologie, il correspond adéquatement à la complexité du sujet. Sur le plan formel, le livre est convenablement pourvu d'un index complet et chaque article d'un bon apparat critique comme d'un résumé concis. Plutôt que de résumer les interventions, ce compte rendu va s'interroger sur l'apport de ce volume à la connaissance et à l'élucidation de la problématique centrale : les rapports entre musique et religion dans l'antiquité. Cette évaluation se centre sur la culture grecque, suivant l'orientation thématique de Kernos.

Le livre est subdivisé selon les trois thématiques développées au colloque. Le premier thème, "Le 'dieu musicien' et son attribut musical », rassemble des contributions sur le panthéon musical (D. Castaldo), sur les mythes touchant aux instruments de musique (la lyre: C. Leduc; l'aulos : Z. Papadopoulou \& V. Pirenne-Delforge) qui sont les attributs des dieux musicaux (Apollon : Ph. Monbrun) auxquels étaient aussi associés des genres de musique traditionnels particuliers (péan : A. Bélis) encore présents dans les compositions plus modernes (comédie : C. Calame). La deuxième partie, « le rituel », est consacrée à la

À propos de Pierre Brulé, Christophe Vendrik̀s (éds), Chanter les dieux: Musique et religion dans l'Antiquité grecque et romaine. Actes du colloque des 16, 17 et 18 décembre 1999, Rennes, Presses Universitaires, 2001 (Collection « Histoire»). 
musique en contexte cultuel. Il s'agit d'abord des rites exécutés en l'honneur des dieux doués de qualités musicales particulières et associés à un type de musique singulier, comme dans les cultes dionysiaques (Th. Menier; G. Sauron). La musique était en outre inséparable de pratiques cultuelles fondamentales comme le sacrifice (J.-R. Jannot; C. Vendries), la prière (Ch. Guittard) et la procession (St. Georgoudi; P. Brulé). La dernière section s'attarde sur « les musiciens du culte » et plus particulièrement sur les aulètes professionnels à l'époque hellénistique (L. Piolot), et leurs pendants romains, les tibicines (V. Péché; J.-M. Pailler) ${ }^{1}$.

Rites, cultes, dieux et mythes sont inséparables, mais la plupart des articles concernent le culte. Ceci correspond aux orientations plus générales de l'enquête scientifique. Ainsí, par exemple, la recherche sur les relations entre médecine et religion se concentre autour des cultes médicaux des dieux guérisseurs. Seulement, le dieu musicien n'a jamais suscité de cultes musicaux au sens strict. En effet, les personnages mythiques aux qualités musicales ne recevaient guère de culte spécifiquement centré sur cette vertu particulière. Or, la musique n'était pas l'apanage des cultes de dieux aux traits musicaux. Tout culte pouvait comporter une composante musicale car la musique appartenait à l'outillage élémentaire de la pratique rituelle. Les qualifications musicales des figures divines ne se manifestaient donc pas tant dans la présence ou l'absence de musique dans leurs cultes, que dans les genres ou types de musique qui y étaient exécutés : les péans, les dithyrambes, etc. Cette musique cultuelle occupe depuis longtemps et de loin la place privilégiée dans la recherche sur les rapports entre musique et religion ${ }^{2}$. Son étude traditionnellement musicologique concerne évidemment plus ses éléments matériels (rythme, tempo, ...) que son référent divin. L'introduction du « dieu musicien » comme objet de recherche est un des grands mérites de ce livre. D'autant plus qu'il se manifeste non seulement dans la musique cultuelle mais davantage encore dans les mythes musicaux, un domaine encore moins exploré de l'interaction entre musique et religion.

Car, dans les mythes musicaux, on ne retrouve point la musique concrète mais sa représentation mythologique. Traditionnellement, c'est toutefois la seule musicologie qui s'occupe de la mythologie à la recherche de la musique. Inversement, en histoire des religions, on s'en tient au contenu mythique des mythes musicaux. L'intervention de Claudine Leduc est exemplaire de ce point de vue. Elle examine le mythe de l'invention de la lyre par Hermès qui la concède à son frère Apollon. Cet échange est traduit dans l'hymne homérique à Hermès par la formule 'cinquante vaches pour une lyre'. Cette opération établit la pbilotês entre les deux frères et elle les pourvoit de leurs parts respectives. Hermès, le nouveau-né, acquiert la houlette qui fait de lui l'bêgêmon du troupeau et le dieu «qui fait crôttre ». Apollon, le frère aîné, reçoit la lyre et devient le maître de la musique, le dernier aspect qui lui manquait. La pbilotês n'est pas fondée sur un simple don mais sur l'amoibè : une transaction commerciale provoquée par Hermès qui se fait du même coup le fondateur de l'échange. Du reste, L. retrouve dans ce mythe des circonstances historiques, des idées philosophiques et des analogies cachées, mais point de musique. Pour L., Hermès se présente essentiellement comme le dieu de l'échange et la lyre comme un instrument d'échange. Ils ont moins à voir avec l'art musical qu'avec la théologie - ainsi le terme de musique n'apparaît que dans le sous-titre : "Musique, échange et théologie dans l'hymne à Hermès I ». Cette interprétation ne clécoule pas tant de la position accessoire de la musique dans cet hymne que de l'orientation surtout mythique de l'analyse. L. s'appuie sur une foule de données et de concepts issus de l'histoire des religions, de la philologie, de la linguistique, de la philosophie et de l'anthropologie, mais elle n'intègre point de dimension musicologique et musicale.

Mieux proportionné et dès lors plus pertinente est l'étude de Zozie Papadopoulou, musicologue, et Vinciane Pirenne-Delforge, historienne des religions, En unissant leurs intérêts, elles examinent la $12^{\star}$ Pythique de Pindare glorifiant l'invention de l'aulétique par Athéna avec la création du nomos polycéphalos à l'occasion de la victoire de Persée sur Méduse, imitant le cri de détresse du monstre et le cri de joie du héros. Par la corrélation

\footnotetext{
1 Le table de matière se trouve dans Kernos 15 (2002), p. 543.

2 Cf. J. QuASTEN, Musik und Gesang in der Kulten der beidnisicben Antike und cbristlicben Frïbzeit, Münster, 1931.
} 
heureuse des aspects philologiques-mythologiques et organologiques-musicologiques, cette étude parvient à clarifier la position problématique de l'aulos dans ce texte, dans la mythologie musicale et dans la culture grecque. Nous apprenons notamment que derrière la formule établie du 'rejet' de l'aulos et du 'triomphe' de la lyre à Athènes au contraire de Thèbes, transparaît en effet une critique de l'aulétique s'opérant dans toute lá culture grecque au cours du $\mathrm{v}^{\mathrm{e}}$ siècle av. J.-C. Elle est également présente dans l'ode du Thébain, notamment dans l'affirmation que l'aulétique est d'origine divine mais subordonnée au chœeur dans des compositions appréciées des dieux. Depuis des temps immémoriaux, les Grecs privilégiaient la parole (le chant) sur la mélodie (l'instrument). Mais les innovations techniques élargissent les possibilités sonores de l'aulos. Les capacités techniques de l'aulète deviennent plus exigeantes, le musicien se professionnalise et l'instrumentiste virtuose arrive au devant de la scène au détriment du chœur et de la musique traditionnelle. Ce n'est pas l'aulos en soi mais les transformations orchestrales provoquées par l'évolution de l'aulétique qui ont compromis la position de cet instrument. La controverse se manifeste dans la mythologie comme dans la philosophie : "le mythe de Marsyas et Apollon dit dans un autre langage que celui de Platon et Aristote les dangers inhérents alux instruments polyphoniques » (p. 54). Dans leur propre langage, les mythes nous renseignent sur la vie musicale des Grecs. Ainsi la polyvalence sonore et la distribution géographique de l'aulos se lisent aussi dans ses associations multiples avec Cybèle, Dionysos, Athéna et même Apollon dans les mythes musicaux. Autant la connaissance du langage mythique que celle des conditions musicales sont indispensables pour les comprendre.

Inversement, les mythes musicaux sont essentiels pour notre compréhension de la musique, de la religion et de leur relation dans l'antiquité grecque. Actuellement, la culture grecque est surtout réputée pour avoir produit les premiers textes littéraires et traités théoriques sur la musique, c'est-à-dire la première musique 'historique'. La musique grecque est devenue l'objet privilégié de l'histoire de la musique, une science renfermée sur la pratique, l'instrumentation, la notation, la théorie et la philosophie de la musique. La musicologie historique approche la musique grecque en tant qu'art tonal. Mais la mousikè technè dépasse largement cette catégorie musicale. Premièrement, il s'agit d'une pratique complexe intégrant à la fois la musique instrumentale, le chant et la danse. Deuxièmement, elle n'est pas une forme d'art autonome mais une activité fondamentalement socio-culturelle et entièrement imbriquée dans la vie religieuse, comme le montrent les mythes musicaux. La musique grecque - toujours selon nos catégories musicologiques - se qualifie plutôt comme 'ethnique'. L'approche plus largement ethnomusicologique peut donc en clarifier maints aspects restés hors d'atteinte de la musicologie historique au sens strict. Ainsi, Philippe Monbrun rapproche la lyre et l'arc, les deux attributs caractéristiques d'Apollon. Ces instruments sont liés par leur matériel, leur construction, leur maniement, etc. Leur relation transcende le temps et l'espace. Le plus ancien chordophone, l'arc musical, est directement associé avec l'arc à flèches. Les Grecs ne connaissaient plus cet instrument primitif sous sa forme matérielle. Mais le lien direct entre la lyre et l'arc est resté fermement ancré dans leur pensée religieuse : «Si Ulysse est un musicien qui bande et accorde son arc comme une phorminx, en joue comme d'une phorminx, l'aède inspiré par Apollon est un archer qui arme sa phorminx comme un arc en direction de la cible, pour décocher ses vers et ses notes comme des flèches » (p. 75). L'arc typique grec était palintonos 'qui se tend en sens inverse', il exige un mouvement palinıropos 'qui se retourne, qui revient sur soi-même', tout comme la lyre. La pensée religieuse des Grecs a donc associé la lyre et l'arc à l'inversion (et à la métis). Leur maniement implique l'harmonisation de tensions bipolaires par des forces opposées et complémentaires. Ainsi ils sont devenus les symboles emblématiques de l'harmonie et de l'opposition en Grèce. Ni l'association de la lyre et de l'arc, ni leur relation avec Apollon ne sont fortuits. Ces associations sont inscrites de façon cohérente et consistante dans ce système de signification qu'est la pensée religieuse des Grecs. Les mythes musicaux sont donc significatifs des conceptions musicales autant que religieuses des Grecs. Cela souligne également l'impertinence de nos propres concepts dans l'étude de la culture grecque.

Notre connaissance de la musique dans la culture grecque est indûment limitée. D'abord par l'emprise exclusive de la musicologie sur l'étude de la musique, ensuite, par l'orientation avant tout historique de la recherche musicologique et, enfin, par le manque cuisant de sources musicales. Aucun son de la musique ancienne n'a évidemment 
survécu. Seuls quelques rares fragments d'instruments et de partitions nous permettent d'essayer de restituer la musique résonante des Grecs ${ }^{1}$. Cette pauvreté de sources est en contraste flagrant avec l'importance effective de l'élément sonore dans la culture grecque. Annie Bélis le démontre à partir des fameux textes avec notation musicale des deux péans inscrits sur le Trésor des Athéniens à Delphes. Le péan est un genre musical que nous étudions généralement comme de la littérature puisqu'il ne nous reste que le texte. Pourtant les paroles étaient choisies en fonction de la mélodie et la mélodie en fonction du rythme, et non l'inverse. Or, le traitement mélodique de textes poétiques (nutbmopoios) pouvait dépasser les règles de la pure scansion par des rythmes et des graphies artificielles, assumant des fonctions expressives et esthétiques. Les qualités musicales ainsi obtenues rendent aux compositions un impact économique et politique. Ces deux péansci étaient destinés à la lutte pour le maintien des privilèges des technites dionysiaques athéniens à Delphes. Leur analyse épigraphique et musico-historique clarifie d'abord des questions de datation, de composition, d'exécution, de notation, d'esthétique, de destination, etc. si chères aux historiens de la musique. Elle relève aussi le caractère musical prééminent du genre du péan, généralement méconnu puisqu'il a si largement perdu sa sonorité et toute sa dimension concrète. L'historienne de la musique insiste également sur le rôle déterminant de l'élément sonore pour le contenu littéraire et les fonctions sociopolitiques du genre musical qu'est le péan. Mais, cette étude de pure musicologie ignore l'impact religieux de l'élément musical du genre religieux qu'est le péan.

Il nous faut un spécialiste comme Claude Calame, toujours sensible à la dimension narrative, énonciative, performative et pragmatique, pour démontrer - dans un vocabulaire souvent ardu pour le lecteur non-francophone - qu'en s'abstenant de la dimension musicale des ouvres littéraires, leur portée religieuse nous est également rendue inaccessible. Une composition doit son impact religieux tant à son texte poétique et à son contenu narratif qu'à sa forme musicale et à son contenu sonore. En l'occurrence, les parties chorales des performances dramatiques n'étaient pas que de simples vers poétiques, mais des vrais chants méliques et de véritables actes de culte : elles sont liées au culte des Muses, elles mettent en scène des actes cultuels imposés par les dieux dans l'action clramatique et elles appartiennent aux actes rituels des festivals dramatiques en l'honneur de Dionysos. Les acteur's du chœur appartiennent à la fois à l'action dramatique qui se joue sur scène et au festival rituel qui se déroule dans le théâtre du sanctuaire de Dionysos. Leur impact religieux atteint son maximum au moment où ces deux fonctions coïncident, comme dans la mise en scène d'un kômos. Le kômos est la composante naturelle des exodoi, lieux privilégiés de l'expression de la réciprocité entre les hommes et les dieux. Dans ses exodoi plus élaborés, Aristophane réalise le passage de l'intra- à l'extradiscursif par le détournement de chants traditionnels liés aux cultes des dieux de l'action sur scène (péans traditionnels, hymnes cultuels, hyménées etc.) en chants de victoire en l'honneur de Dionysos, le dieu des concours rituels dans lesquels les pièces dramatiques espéraient remporter la victoire. Cette transformation est effectuée par l'utilisation de pronoms, de temps, de rythmes et d'autres: "moyens musicaux d'une 'ritualisation' du second degré qui transforme l'issue heureuse de l'intrigue fictionnelle représentée dramatiquement sur la scène en une victoire au concours dramatique célébré en l'honneur de Dionysos »(p. 137). Cette étude démontre également l'entrelacement foisonnant des rites, des cultes, des dieux et des mythes, et la haute complexité de leur interrelation avec la musique : les textes, s'agissant des mythes et des dieux musicaux, assumaient la forme de performances musicales exécutées en l'honneur même des dieux musicaux.

À coté des textes, l'iconographie est une des sources principales des sciences historiques. Dans leur propre langage, les images nous livrent des données de contenu souvent différent et de valeur toujours complémentaire à celles des textes. Daniela Castaldo le montre au sujet des principaux dieux musiciens et leurs instruments musicaux. Contrairement à l'association rigide des chordophones avec l'apollinien et des aérophones avec le dionysiaque, qu'on retrouve dans les textes, les vases représentent le barbitos, par exemple, comme un instrument essentiellement dionysiaque, accessoire 
typique du symposium, des chants de banquet, les danses du kômos, etc. Même la lyre est quelques fois associée à la consommation du vin par les satyres et Dionysos. Le caractère des scènes picturales n'est pas univoquement déterminé par les instruments de musique qui y sont représentés. Le même instrument évoque un tout autre genre de musique dans une scène mouvementée que dans un contexte tranquille. Ceci correspond à la théorie (mimétique) que l'audience reproduit le rythme et participe de la musique par ses mouvements du corps. Si la musique n'est plus audible, elle est néanmoins encore perceptible. Cette étude démontre que l'institution de l'iconographie musicale dans l'histoire de la musique comme une branche autonome par rapport à l'historiographie musicale est pertinente. Par contre, elle confirme l'obsession de l'histoire de la musique dans la recherche de la musique réelle et résonante, ce qui est fait au plus grand détriment du caractère mythique des images. Cette étude ne parvient pas à la mythologie et à l'histoire des religions. Elle s'en tient à la musique et à l'histoire. C'est trop réducteur. Les mythes musicaux étaient une composante fondamentale de la vie musicale comme de la vie religieuse. Ce sont des témoins directs de leur enchevêtrement, incitant à une approche plus intégrée et globale dans l'étude de la musique et de la religion dans l'antiquité.

Leur interrelation se manifeste dans la mythologie comme dans le culte où elle est plus concrètement observable, empiriquement accessible et donc repérable par les sciences historiques. La coïncidence des pratiques musicales et cultuelles dans des processions, des concours, des offrandes, etc. représente la composante la plus évidente de l'interaction entre la musique et la religion pour l'historien de la musique comme pour l'historien de la religion grecque, par exemple à travers les nombreux documents cultuels mentionnant des pratiques musicales. Ainsi, Stella Georgoudi rencontre le règlement cultuel de la procession chantante exécutée par les Molpes, une confrérie liée au culte d'Apollon Delphinios, le dieu poliade de Milet. Contrairement aux idées reçues, les Molpes ne sont pas de simples chanteurs. G. démontre que le verbe melpô n'est pas un synonyme d'aidô (et que le substantif molpê n'équivaut pas à aiodê). Melpô, melpestai et molpê renvoient à une célébration plus largement musicale, à un ensemble de mélodies et de mouvements rythmiques. $G$. clémontre que cette famille de mots a le double sens de chanter et de danser, tout comme Apollon se montre le dieu du chant et du mouvement. Les Molpes de Milet, dignes donc de leur nom et de leur patron, étaient réputés organiser une procession annuelle du sanctuaire d'Apollon à Milet jusqu'à celui de Didymes. Cette célébration en cortège était ponctuée par sept arrêts pour chanter et danser un péan en l'honneur d'Apollon et d'autres divinités qui lui étaient liées (Hermès, les Nymphes,...). La procession se présente comme un événement fort complexe comprenant toute une série d'actes rituels (chant, danse, célébrations,...), tout un ensemble de figures divines et héroïques (Apollon, Hécate, Hermès,...) et même un passage physique et psychique d'un Apollon, celui de Milet, à un autre, l'Apollon oraculaire de Didymes. La musique était l'un des moyens instrumentaux de ce procès comme de cette procession.

La musique assumait également une fonction instrumentale dans l'induction de la transe et de l'enthousiasme, ces expériences fondatrices de la religiosité dionysiaque. Les sources manifestent une prédominance frappante, mais pas tout à fait exclusive, de la musique de l'aulos dans les contextes dionysiaques. Si la musique incite à la transe, constate Thomas Menier, ce n'est donc pas par l'effet sui generis de la substance sonore propre à tel ou tel instrument. Aristote le confirme : ce sont les mélodies d'Olympos et la musique du mode phrygien qui rendent les hommes enthousiastes (Pol. 1340a). Le transport dionysiaque trouve son expression principale dans l'aulos puisqu'il convient le mieux au mode phrygien. Ce mode s'oppose au mode dorien, qui se sert de préférence de la lyre. Apparemment, ces deux systèmes concurrents existent toujours en Grèce. L'un est pentatonique et sans demi-ton, l'autre est diatonique c'est-à-dire avec demi-ton. Le mode jouant sur l'opposition ton et demi-ton obtient la plus grande portée et expressivité. On l'a assimilé au mode phrygien qui est réputé pour sa charge affective, son expressivité mimétique et son très vaste champ d'application. Le mode phrygien convient le mieux aux émotions vigoureuses suscitées par le rituel dionysiaque, mais toutes les mélodies de l'aulos dans le mode phrygien ne lui convenaient pas pour autant. Les mélodies d'Olympos et de Marsyas s'y adaptent singulièrement. Leur spécificité ne peut se situer au niveau de leur forme (mode) ou de leur contenu musical (instrument). Elle doit se trouver dans le domaine extra-musical ou symbolique. $M$. estime qu'il pourait s'agir des pièces musi- 
cales pour aulos provenant de la Phrygie même. Ainsi l'efficacité dionysiaque des pièces musicales ne résiderait pas tant dans leurs qualités physiques ou intra-musicales, que dans leur étrangeté, leur descendance de Phrygie, leur rapport direct avec Dionysos, d'où leur aptitude à le manifester, à le symboliser. Ils sont le véhicule privilégié de l'enthousiasme dionysiaque puisqu'ils sont eux-mêmes littéralement 'pleins du dieu'.

Les qualités extra-musicales ou symboliques de la musique peuvent bien entendu être perçues au sein même des données 'historiques' (temps-espace), mais elles le sont encore davantage dans la sphère du divin. Il nous faut un historien des religions comme Pierre Brulé, toujours attentif à s'élever au-dessus du 'plancher des vaches', pour soulever le problème. Il démontre que dans la musique s'unissent les hommes et les dieux, tout comme dans le gamos. Dans la musique du gamos, comme dans tout rituel, la musique instrumentale (mélodie) s'accouple à la poésie (parole) et à la danse (mouvement). La célébration du gamos est un spectacle complet de cris, de musique, de lumière, de torches, de ballet, de chours, etc. entamant l'ouie et la vue, invoqués par les formules 'aneche, pareche' : lève ta torche, éclaire-moi, et 'bymen ô byménée', chante l'hyménée, l'hymne en l'honneur' d'Hyménée, le héros du mariage. Son nom trouve son origine d'abord dans la pratique rituelle des cris réitérés marquant le rituel du gamos, puis dans la musicalité recherchée au moyen des formulations proclamées, et enfin dans l'antropomorphisation et la divinisation des formules musicales. Cette succession correspond à la centralité du rythme dans la musicalité grecque. C'est du cri qu'est né Hyménée, et non l'inverse. L'hyménée est une pratique rituelle et un personnage mythique. Son trait caractéristique est d'aimer et d'être aimé. Il est jeune et beau comme une fille, tant et si bien qu'on le prend souvent lui-même pour une fille. Ce beau jeune efféminé est également un musicien merveilleux sur la cithare, son instrument préféré. Les citharèdes sont réputés "avoir l'âme faible » (Platon, Symp. 176d). Hyménée, aimé par ses maîtres, aime les garçons. Il est craint par les pères parce qu'il entrave la production d'enfants légitimes. Loin de répondre à la réalité de l'époux grec, il incarne le garçon amant qui meurt lors du mariage. Cette tragédie est l'aition de l'hyménée, chant de mariage et chant funèbre à la fois. La musique est l'accompagnement typique de ces événements extrêmement émotionnels. Dans l'exécution de la cérémonie nuptiale, l'élément sonore prend la forme d'une orchestique antagoniste avec deux chours opposés interprétant la position de chacun des sexes. Ils chantent la nympbè et ses craintes comme le nympbios et ses impatiences. Ces joutes chorales matrimoniales intègrent les filles et les garçons, la musique vocale et instrumentale, la musique et la danse. Car la danse et le chant apportent davantage à la beauté des jeunes, à leur clésirabilité. Ils leur rendent leur charis, les rapprochent du divin. Dans la performance des choeurs du gamos, le présent humain et l'au-delà divin coincident temporairement, comme aux noces de Cadmos et d'Harmonie où les mortels reçoivent comme cadeaux nuptiaux la lyre d'Hermès, l'aulos d'Athéna, la citharodie d'Apollon et l'aulodie des Muses. Lá musique humaine est un don divin qui en écho à la musique divine, réunit l'humain au divin. La musique est du côté des dieux avant d'être du côté des hommes. Par la belle musique surtout, les hommes peuvent se rapprocher des dieux.

Pierre Brulé ne se contente pas simplement de constater que les Grecs ont conçu la musique comme une partie intégrante du divin. Dans la préface tout à fait originale qu'il livre d'entrée de jeu, il s'interroge sur ce point. Cette enquête l'amène à observer que la musique et le divin sont spécialement liés par leur indicibilité. Le vocabulaire musicologique avec ses termes techniques comme rythme, timbre, tempo, etc. ne parvient pas à traduire les émotions induites par la musique. L'invocation du lexique d'autres sens (la vue, le sens, ...) ou le recours aux psychologismes primitifs (triste, gai, ...) restent nos principaux moyens d'approximation des affects musicaux. Le caractère énigmatique de la musique se mesure par la pauvreté de son lexique et encore plus clairement par l'attention portée à la question de ses origines. Le mystère ontologique et épistémologique que représente la musique est généralement réduit à l'énigme de ses origines. L'obsession des origines caractérise autant l'étude de la musique que l'étude des religions. Pour les Grecs, la musique était évidemment d'origine et de nature divine, les mythes musicaux le confirment. En effet, la musique est assimilée à une sorte de langage permettant la communication entre les hommes et les dieux car elle est produite par les êtres humains mais de nature divine. Dans la mythologie, la musique intervient aussi dans les relations entre 
les dieux et, dans le culte, elle met les hommes en contact. La musique agit sur les groupes sociaux comme sur l'individu et son effet se situe dans le domaine cognitif, affectif comme psychomoteur. Ainsi, ce phénomène physique et biologique qu'est la musique s'opère au niveau psychologique comme sociologique et s'élève finalement au domaine religieux. Elle agit comme une sorte de 'sur-langage', le langage des immortels, que les hommes tentent d'approcher, d'imiter avec leurs instruments. La musique humaine est un moyen singulièrement approprié de se rapprocher des dieux, de dire le divin indicible. La consubstantialité du langage musical et du divin se matérialise évidemment dans l'étroitesse et la complexité de l'interrelation entre musique et religion, aussi bien dans les mythes que dans les cultes de l'antiquité. Ainsi, les compositions musicales (hymnes, péans, comédies, ...) étalient une constituante typique des pratiques religieuses (concours, festivals, processions, prières,...). Ils étaient entièrement consacrés aux dieux et héros dont ils traitaient (mythes musicaux) et en l'honneur desquels ils étaient exécutés (musique cultuelle). Laurent Piolot insiste d'ailleurs sur l'impossibilité de distinguer une musique 'religieuse' et une musique 'profane'. Autant la musique que la religion étaient complètement imbriquées dans toute la vie socio-culturelle. L'une n'y va pas sans l'autre. Cette interrelation dépasse le niveau purement matériel et même fonctionnel; elle a un sens plus profond. Ce n'est pas un simple hasard si la nature du divin pensée par les Grecs correspond étroitement à la conception grecque de la musique. Il est également impossible de saisir cette ampleur sans le double regard musical et religieux, dont la pertinence va loin au-delà de ce livre et certainement de ce résumé. Mais nous avons tout de même pu constater que ce n'est pas du tout un hasard si les Grecs ont " chanté leurs dieux »!

Vrije Universiteit Brussel

Centrum Leo Apostel

Pleinlaan 2

$\mathrm{B}-1050$ Brusset. 